\title{
BIOPROSPECCIÓN MODULADA POR LA FENOLOGÍA, EL SEXO Y EL GRADIENTE ALTITUDINAL: EL CASO DE BACCHARIS CONFERTA SOBRE MYROTHECIUM RORIDUM Y ALTERNARIA SOLANI BIOPROSPECTING MODULATED BY PHENOLOGY, SEX AND ALTITUDINAL GRADIENT: THE CASE OF BACCHARIS CONFERTA ON MYROTHECIUM RORIDUM AND ALTERNARIA SOLANI
}

\author{
@María Guadalupe Ruiz-Gómez¹, @Francisco Abelardo Cen-Pacheco², @María del Rosario Pineda-López¹,

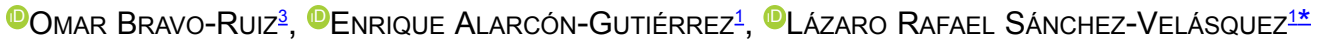

${ }^{1}$ Instituto de Biotecnología y Ecología Aplicada INBIOTECA, Universidad Veracruzana, Xalapa, Veracruz, México.

${ }^{2}$ Facultad de Bioanálisis-Veracruz Boca del Río, Universidad Veracruzana, Veracruz, México.

'Laboratorio de Análisis Clínicos Carlin, Veracruz, México.

*Autor de correspondencia: lasanchez@uv.mx

\section{Resumen}

Antecedentes: La bioprospección incluye la búsqueda de metabolitos secundarios para ser usados como fármacos, fungicidas o insecticidas. Los metabolitos secundarios en plantas pueden variar de acuerdo con su estado fenológico, sexo y por el ambiente, por lo tanto, estos elementos deben ser considerados en la bioprospección.

Preguntas: 1) ¿Son diferentes las fenofases de Baccharis conferta entre organismos de diferente sexo y a lo largo de un gradiente altitudinal? y 2) ¿Tienen actividad biológica las diferentes concentraciones de extractos etanólicos de diferente sexo, fenofases y altitudes de $B$. conferta sobre el crecimiento in vitro de Myrothecium roridum y Alternaria solani?

Especies estudiadas: Baccharis conferta Kunth, Myrothecium roridum Tode ex Fr. y Alternaria solani Sor.

Sitio de estudio y fechas: Parque Nacional Cofre de Perote, septiembre 2017 a octubre 2018.

Métodos: Registro mensual de fenofases en plantas de ambos sexos de $B$. conferta en tres altitudes del Cofre de Perote. Actividad antifúngica in vitro de extractos de $B$. conferta sobre Myrothecium roridum y Alternaria solani.

Resultados: Observamos diferencias $(p<0.05)$ en la presencia y duración de las fenofases de $B$. conferta entre sexos y altitudes. También hubo diferencias significativas de los extractos provenientes de organismos de diferentes altitudes y fenofases sobre el crecimiento de M. roridum y $A$. solani.

Conclusiones: Las fenofases de Baccharis conferta son diferentes entre sexos y a través de la altitud, además sus extractos (entre sexos y altitud) tienen efectos diferenciales sobre el crecimiento in vitro de Myrothecium roridum y Alternaria solani.

Palabras clave: Actividad antifúngica, fenología, México, Parque Nacional Cofre de Perote, roya, tizón temprano.

\begin{abstract}
Background: Bioprospecting includes the search for secondary metabolites to be used as drugs, fungicides or insecticides. Secondary metabolites in plants may vary according to their phenological stage, sex and environment, therefore, these elements should be considered in bioprospecting.

Questions: 1) Are the Baccharis conferta phenophases different between organisms of different sexes and along an altitudinal gradient? and, 2) Do the different concentrations of ethanolic extracts of different sex, phenophases and altitudes of $B$. conferta on the in vitro growth of Myrothecium roridum and Alternaria solani have biological activity?

Species studied: Baccharis conferta Kunth, Myrothecium roridum Tode ex Fr. and Alternaria solani Sor.

Study site and dates: Cofre de Perote National Park, September 2017 to October 2018.

Methods: Monthly registration of phenophases in plants of both sexes of B. conferta in three altitudes of the Cofre de Perote. In vitro antifungal activity of extracts of B. conferta on Myrothecium roridum and Alternaria solani.

Results: We observed differences $(p<0.05)$ in the presence and duration of the B. conferta phenophases between sexes and altitudes. There were also significant differences in extracts from organisms of different altitudes and phenophases on the growth of M. roridum and A. solani. Conclusions: The phenophases of Baccharis conferta are different between sexes and through altitude, and its extracts (between sexes and altitude) also have differential effects on in vitro growth of Myrothecium roridum and Alternaria solani.
\end{abstract}

Keywords: Antifungal activity, Cofre de Perote National Park, early blight, phenology, Mexico, rust.

Este artículo se encuentra bajo los términos de la licencia Creative Commons Attribution License CCBY-NC (4.0) internacional. 
Los estudios de bioprospección en plantas son básicos para la búsqueda de metabolitos secundarios útiles en el control de enfermedades humanas y de plagas en cultivos agrícolas (Strobel \& Daisy 2003, Ruiz-Bustos et al. 2009). Sin embargo, la bioprospección en plantas generalmente se realiza sin considerar los procesos fenológicos (v.gr., Peraza-Sánchez et al. 2007, Funes et al. 2018), y ello puede conducir a cometer omisiones importantes en el muestreo y subestimar o sobrestimar su potencial de uso. No obstante, también existen trabajos que integran algunas fases fenológicas a los estudios de bioprospección (v.gr., Elmqvist et al. 1991, Feresin et al. 2000, Masotti et al. 2003, FloresEstévez et al. 2013).

La fenología es una subdisciplina de la ecología que estudia la temporalidad de los eventos biológicos cíclicos y la relación de estos con factores bióticos y abióticos (Williams-Linera \& Meave 2002). Los eventos fenológicos son conocidos como fenofases, los cuales en las plantas se dividen en vegetativas y reproductivas, en este sentido podemos hablar de fenología vegetativa y fenología reproductiva. La fenología está estrechamente relacionada con los factores abióticos y bióticos. Se han realizado estudios que asocian la altitud y la fenología de plantas en regiones montañosas, los cuales señalan cambios en la fenología reproductiva (Ziello et al. 2009) y en la composición química de la madera de especies arbóreas (Musule et al. 2016). Por ejemplo, en algunas especies arbustivas, se ha observado asincronía en la floración relacionada con la altitud (y ésta con la temperatura y la precipitación), ya sea que ocurra primero en las zonas más bajas que en las altas o viceversa, como ocurre en Erica arborea L. y Calluna vulgaris (L.) Hull, respectivamente (Vera 1995). Además, esto se puede complejizar en organismos dioicos, como algunas especies del género Baccharis, en las cuales puede diferir la floración entre sexos. Por ejemplo, en B. dracunculifolia DC. los organismos masculinos producen mayor cantidad de inflorescencias y de manera más temprana que los femeninos (Espírito-Santo et al. 2003). Además, en B. concinna, G. M. Barroso reportó mayor cantidad de organismos femeninos en zonas bajas respecto a las zonas más altas (Marques et al. 2008). Por lo que sigue siendo un desafío comprender el comportamiento de este género en un gradiente altitudinal.

Los metabolitos secundarios de las plantas, los cuales les confieren actividades biológicas, difieren en los organismos a lo largo de su ciclo fenológico (Elmqvist et al. 1991) y este proceso varía con la altitud (Ziello et al. 2009), consecuentemente los metabolitos secundarios pueden fluctuar o pueden estar presentes o no en poblaciones de la misma especie a distintas altitudes. Lo anterior debe tenerse presente para la extracción de metabolitos secundarios en plantas.
El género Baccharis ha sido motivo de estudios de bioprospección, debido a que diversas especies de este género son utilizadas en la medicina tradicional para mitigar enfermedades como anemias, diabetes y enfermedades de órganos como el estómago, el hígado y la próstata (Verdi et al. 2005). Hasta el momento se han identificado más de 150 compuestos en el género Baccharis y sus estudios farmacológicos se basan principalmente en sus propiedades antiinflamatorias, antioxidantes, antimicrobianas y antifúngicas (Abad \& Bermejo 2007). Algunas de las especies de este género que se reportan con actividad antifúngica son: B. darwinii Hook. \& Arn. con actividad fungicida contra Microsporum gypseum (E.Bodin) Guiart \& Grigoraki, Trichophyton rubrum (Castell.) Sabour., Trichophyton mentagrophytes (Robin) Blanchard y Cryptococcus neoformans (Sanfelice) Vuillemin, (Kurdelas et al. 2010); inhibiendo el crecimiento como B. glutinosa Pers. en Fusarium verticillioides (Sacc.) Nirenberg, Aspergillus flavus Link y Aspergillus parasiticus Speare (Rosas-Burgos et al. 2009), B. dracunculifolia sobre Candida krusei (Castellani) Berkhout y Cryptococcus neoformans (Da Silva et al. 2008) y B. pedunculata (Mill.) Cabrera en hongos patógenos humanos (Rahalison et al. 1995).

Sin embargo, tanto para especies de este género como para otras, sólo se han tomado muestras de una altitud y no se describen las fases fenológicas dentro de las cuales fueron colectadas para la bioprospección. Esto puede ocasionar una sobreestimación o subestimación de la cantidad y presencia de los metabolitos secundarios en las plantas, de tal manera que se puede exagerar o desvalorar su potencial.

En el presente estudio se utilizaron, como modelo, individuos de Baccharis conferta Kunth, de diferentes sexos, fenofases y altitudes, para explorar la presencia de metabolitos secundarios contra el crecimiento in vitro del hongo Myrothecium roridum Tode ex Fr., que causa necrosis foliar a plántulas como el café y la soya (Silva et al. 2014, Haudenshield et al. 2018) y del hongo Alternaria solani Sor., que causa tizón temprano en papa. El objetivo de este trabajo es demostrar la importancia de las fases fenológicas, el sexo y la altitud de las plantas en los procesos de bioprospección. Las preguntas que nos hicimos en este trabajo fueron: 1) ¿Son diferentes las fenofases vegetativas y reproductivas de Baccharis conferta entre organismos de diferente sexo? 2) ¿Son diferentes las fenofases vegetativas y reproductivas de $B$. conferta a lo largo de un gradiente altitudinal? y 3) ¿Tienen actividad biológica las diferentes concentraciones de extractos etanólicos de diferente sexo, fenofase y altitud de B. conferta sobre el crecimiento in vitro de Myrothecium roridum y Alternaria solani? 


\section{Materiales y métodos}

Organismos estudiados. Baccharis conferta es un arbusto erecto o semipostrado, glutinoso y mide aproximadamente hasta $2.7 \mathrm{~m}$ de alto, tanto sus tallos, ramas y ramillas son glabros. Es una especie dioica y muestra diferencias claras entre sus flores masculinas y femeninas (Calderón de Rzedowski \& Rzedowski 2005). B. conferta puede fungir como nodriza de Abies religiosa (Kunth) Schltdl. \& Cham (Sánchez-Velásquez et al. 2011), además es utilizada en la medicina tradicional para aliviar dolores de estómago en las comunidades del Cofre de Perote, como laxante, para estimular la micción o para perder peso (Abad \& Bermejo 2007). En el centro de Veracruz, México, es comúnmente conocida por el uso que se le da para hacer escobas rústicas. Hasta el momento existen dos estudios donde reportan los meses en que $B$. conferta presenta su floración y fructificación en un solo sitio (Vázquez 2014, CornejoTenorio \& Ibarra-Manríquez 2007) y aún se desconoce su fenología anual y las variaciones fenológicas a lo largo de un gradiente altitudinal. Tampoco se conoce su potencial antifúngico sobre $M$. roridum y $A$. solani.

Myrothecium roridum (Familia: Incertae sedis) es un hongo fitopatógeno de distribución cosmopolita con más de 200 especies de plantas hospederas de diferentes familias, entre ellas se incluyen ornamentales, hortícolas y frutales (Cabral et al. 2009), principalmente, el tomate, la soya, el café y el algodón (Conrado et al. 2006, Fernando et al. 1986). Es considerado como un habitante del suelo asociado a materia orgánica en descomposición, además se ha demostrado que $M$. roridum está ampliamente distribuido en viveros (Bunster \& Torres 2003). Las esporas de M. roridum pasan gran parte de su ciclo de vida en el suelo (Fernando et al. 1986). Posteriormente, infectan a las plantas por medio de conidióforos, los cuales ocasionan síntomas como el estrangulamiento en el tallo. También se ha observado que existe abundante esporulación en los tallos de las plantas, dichas esporas pueden dispersarse eficientemente a través del riego por aspersión en condiciones normales de invernadero o por acción del viento en los campos de cultivo (Bunster \& Torres 2003). En hojas causa clorosis que con el paso del tiempo se convierten en lesiones necróticas que se observan de forma circular de color café oscuro, y cuando las lesiones envejecen se forma un agujero por la acción de la humedad (Fish et al. 2012). En las semillas depositadas en el suelo pueden producir necrosis, además de la presencia de toxinas y enzimas producidas por el hongo, involucradas en la inhibición de la germinación de semillas. El crecimiento de $M$. roridum en medios de cultivo se observa con zonas concéntricas de micelio blanquecino y la esporulación inicialmente es de color verde oscuro, que más adelante se cubre con círculos negros en los que se contienen los conidios (Da Silveira et al. 2007).
Alternaria solani, es un hongo fitopatógeno de la familia Pleosporaceae que presenta variabilidad morfológica y patogénica entre sus aislados. Este hongo afecta diversos cultivos como la papa y el tomate ocasionando necrosis en hojas y en tubérculos de la papa. Está ampliamente distribuido en el planeta en áreas con condiciones climáticas favorables de alta humedad y baja temperatura (Graves 1996). Cultivado in vitro produce una colonia filamentosa de color negra profundamente pigmentada con una capa blanca, aunque en laboratorio hemos observado que su aspecto cambia dependiendo del medio y la temperatura a la que se mantenga. El medio óptimo para su desarrollo es PDA (Potato Dextrose Agar; Kumar et al. 2008). Visto al microscopio, A. solani se presenta en forma de conidios asexuales y se pueden encontrar solos o en pares. Poseen entre 9 y 11 septos transversales cada uno, de los cuales el último se caracteriza por presentar un alargamiento.

Sitio de estudio. El estudio fenológico se llevó a cabo en el Parque Nacional Cofre de Perote (PNCP), Veracruz, México $\left(19^{\circ} 25^{\prime} 33^{\prime \prime}\right.$ a $19^{\circ} 33^{\prime} 52^{\prime \prime}$ de latitud norte y entre los meridianos de $97^{\circ} 06^{\prime} 55^{\prime \prime}$ a $97^{\circ} 12^{\prime} 52^{\prime \prime}$ de longitud oeste). Está ubicado entre el cinturón Volcánico Transmexicano y el extremo sur de la Sierra Madre Oriental. Su topografía es accidentada, el intervalo altitudinal va de 3,000 a $4,250 \mathrm{~m} \mathrm{snm}$ e incluye cinco tipos de climas (semifrío húmedo, semifrío subhúmedo, templado húmedo, templado subhúmedo y frío subhúmedo) y suelos (Andosol y Regosol eútrico) (SEMARNAT 2015), que dan como resultado una amplia gama de tipos de vegetación fríos y secos en las zonas altas y cálido-húmedos en las zonas bajas (CarvajalHernández \& Krömer 2015). Los tipos de vegetación dentro del PNCP son bosque caducifolio, bosque de PinusQuercus, bosque de Pinus, bosque de Abies, páramo de altura y pastizales (Rzedowski 1978). B. conferta es típica de la vegetación secundaria (primeros estados sucesionales) de bosques de coníferas.

Muestreo fenológico. En la ladera norte del PNCP, se seleccionaron tres sitios con presencia de B. conferta a lo largo de un gradiente altitudinal $(2,900,3,450$ y $3,670 \mathrm{~m} \mathrm{snm}$ ) con una exposición solar y pendientes similares (Tabla 1). Los individuos seleccionados $(\mathrm{n}=70)$ fueron etiquetados por sexo (35 masculinos y 35 femeninos) en época de floración y se llevaron a cabo visitas mensuales a lo largo de un año (de septiembre 2017 a octubre 2018) para registrar la presencia o ausencia de las fenofases en la que se encontraban, es decir producción de brotes (hojas nuevas en las ramas) Figura $1 \mathrm{~A}$, y la fase reproductiva (preantesis con corola no visible, Figura 1B; antesis masculina y femenina con corola visible, Figuras $1 \mathrm{C}$ y D, respectivamente; producción de semillas y senescencia con corona senescente o caída (Figuras 1E y $\mathrm{F}$, respectivamente). El número de individuos fue menor en el sitio tres $(\mathrm{n}=10$, ubicado a mayor altitud), debido a la escasez de individuos. 
Tabla 1. Ubicación de los sitios estudiados de Baccharis conferta en diferentes altitudes en la ladera norte del Parque Nacional Cofre de Perote, Veracruz, México.

\begin{tabular}{|c|c|c|c|c|c|}
\hline Sitio & Altitud (m snm) & Exposición & Pendiente & Coordenadas & Número de individuos observados \\
\hline \multirow[t]{2}{*}{1} & 2,900 & Noroeste & $2.7^{\circ}$ & N $19^{\circ} 34^{\prime} 23.1^{\prime \prime}$ & 30 \\
\hline & & & & O $97^{\circ} 07^{\prime} 59.3^{\prime \prime}$ & \\
\hline \multirow[t]{2}{*}{2} & 3,450 & Norte & $13^{\circ}$ & N 193 $31^{\prime} 16.2^{\prime \prime}$ & 30 \\
\hline & & & & O $97^{\circ} 09^{\prime} 37.7^{\prime \prime}$ & \\
\hline \multirow[t]{2}{*}{3} & 3,670 & Noroeste & $7^{\circ}$ & N $19^{\circ} 30^{\prime} 47.8^{\prime \prime}$ & 10 \\
\hline & & & & O $97^{\circ} 09^{\prime} 46.5^{\prime \prime}$ & \\
\hline
\end{tabular}

Relación entre fenología, altitud, sexo y bioprospección exploratoria in vitro. Para la exploración in vitro, se llevó a cabo la colecta de $B$. conferta en los mismos individuos observados para la fenología y se hizo sólo en los sitios donde había mayor número de individuos (sitio uno y dos), en el sitio tres no encontramos suficientes individuos para la colecta de material para la extracción etanólica (Tabla 1). En cada uno de estos dos sitios (2,900 y 3,450 m snm) se eligieron aleatoriamente sólo 20 organismos sanos (10 de cada sexo, $\mathrm{n}=2$ ) que medían entre 1.5 y $2 \mathrm{~m}$ de altura, y a través del tiempo se colectó el material de las fenofases (produciendo brotes y antesis), de ambos sexos. Se cortaron con tijeras las partes extremas de las ramas con brotes o inflorescencias (según la fenofase de los organismos, $\mathrm{n}=2$ ) los brotes se colectaron en octubre 2017 en ambos sitios $(2,900$ y $3,450 \mathrm{~m} \mathrm{snm})$ y las inflorescencias en febrero 2018 en el sitio $2(3,450 \mathrm{~m}$ snm) y en marzo 2018 en el sitio 1 $(2,900 \mathrm{~m} \mathrm{snm})$, y se introdujeron en pequeños sacos de manta de algodón separando las muestras por sexo, hasta obtener un aproximado de $1 \mathrm{~kg}$ de material fresco por muestra, obteniendo un total de 8 muestras, de las cuales se prepararon extractos para el estudio in vitro (Tablas 2 y $\underline{3}$ ). El material fresco se trasladó al laboratorio del Instituto de Biotecnología y Ecología Aplicada (INBIOTECA) de la Universidad Veracruzana, para ser deshidratado a $40{ }^{\circ} \mathrm{C}$ hasta obtener un peso seco constante.

Una vez deshidratadas las muestras para cada periodo de colecta, el material se trituró para realizar extractos etanólicos al $96 \%$ de cada muestra, colocando $1 \mathrm{~L}$ de etanol por cada $250 \mathrm{~g}$ de material vegetal seco colectado en brotes y $1 \mathrm{~L}$ de etanol por cada $150 \mathrm{~g}$ de material vegetal seco colectado en floración, ya que este último es más voluminoso. Posteriormente se dejó reposar siete días en total oscuridad a temperatura ambiente y después, el etanol se filtró utilizando papel filtro Whatman número 5. A continuación se procedió a colocar nuevamente etanol al $96 \%$ hasta cubrir la muestra y se dejó reposar nuevamente siete días en total oscuridad a temperatura ambiente y se filtró nuevamente el etanol. Finalmente, los extractos obtenidos se sometieron a evaporación rotatoria bajo presión reducida, utilizando un Rotavapor R-3 equipado con una bomba de vacío V-700 a una presión de $175 \mathrm{mbar}$ (Büchi, Latinoamérica S. de R.L. de C.V.) y un baño maría a una temperatura de $40{ }^{\circ} \mathrm{C}$. Las muestras concentradas se almacenaron en refrigerador $5 \pm 2{ }^{\circ} \mathrm{C}$ hasta establecer los experimentos simultaneamente (Angulo-Escalante et al. $\underline{2009)}$.

Myrothecium roridum fue obtenido de una cepa preservada en el cepario del laboratorio de Ecología del INBIOTECA. La cepa fue reactivada en cajas Petri con medio V8-Agar mediante la inoculación de discos de agar de $6 \mathrm{~mm}$ de diámetro, el cual contenía el micelio del hongo. Una vez reactivada, se inocularon cajas con medio V8-Agar y ampicilina $(35 \mu \mathrm{g} / \mu \mathrm{L})$ tomando una espora con la punta de una aguja y sembrándola por punción en el centro de cada caja Petri de $5 \mathrm{~cm}$ de diámetro. Inmediatamente se aplicaron, a una distancia de $2 \mathrm{~cm}$ de la espora y directamente sobre el medio V8-Agar, los siguientes tratamientos; extractos etanólicos (concentraciones de 25, 15 y $10 \mathrm{mg}$ ) provenientes de individuos crecidos a dos altitudes (2,900 y 3,450 m snm), de dos fenofases (antesis y producción de brotes) y diferente sexo (masculino y femenino); con excepción del tratamiento de extracto de organismos masculinos con brotes de una altitud de 3,450 m (no se obtuvo extracto suficiente para el experimento). Además, se aplicó un testigo control positivo de $0.0125 \mu \mathrm{g}$ de ingrediente activo de fungicida carbendazim (nombre comercial Prozycar, Promotora Técnica Industrial, S.A de C.V.) y un testigo control negativo $10 \mu \mathrm{L}$ de etanol al $96 \%$ (Tabla 2).

En una caja Petri se colocaron tres concentraciones de extractos etanólicos con sus respectivos testigos, para cada altitud, fenofase y sexo. Para cada grupo de tratamiento se usaron tres repeticiones. Como referencia de crecimiento del hongo, se colocó una espora en tres cajas Petri. El cultivo se sometió a incubación por 7 días a $28^{\circ} \mathrm{C}$ en una incubadora (marca Binder, USA), la variable de respuesta fue, como inhibición, la distancia entre el crecimiento micelial y los tratamientos, o en su caso, la invasión o no del micelio sobre los tratamientos. 

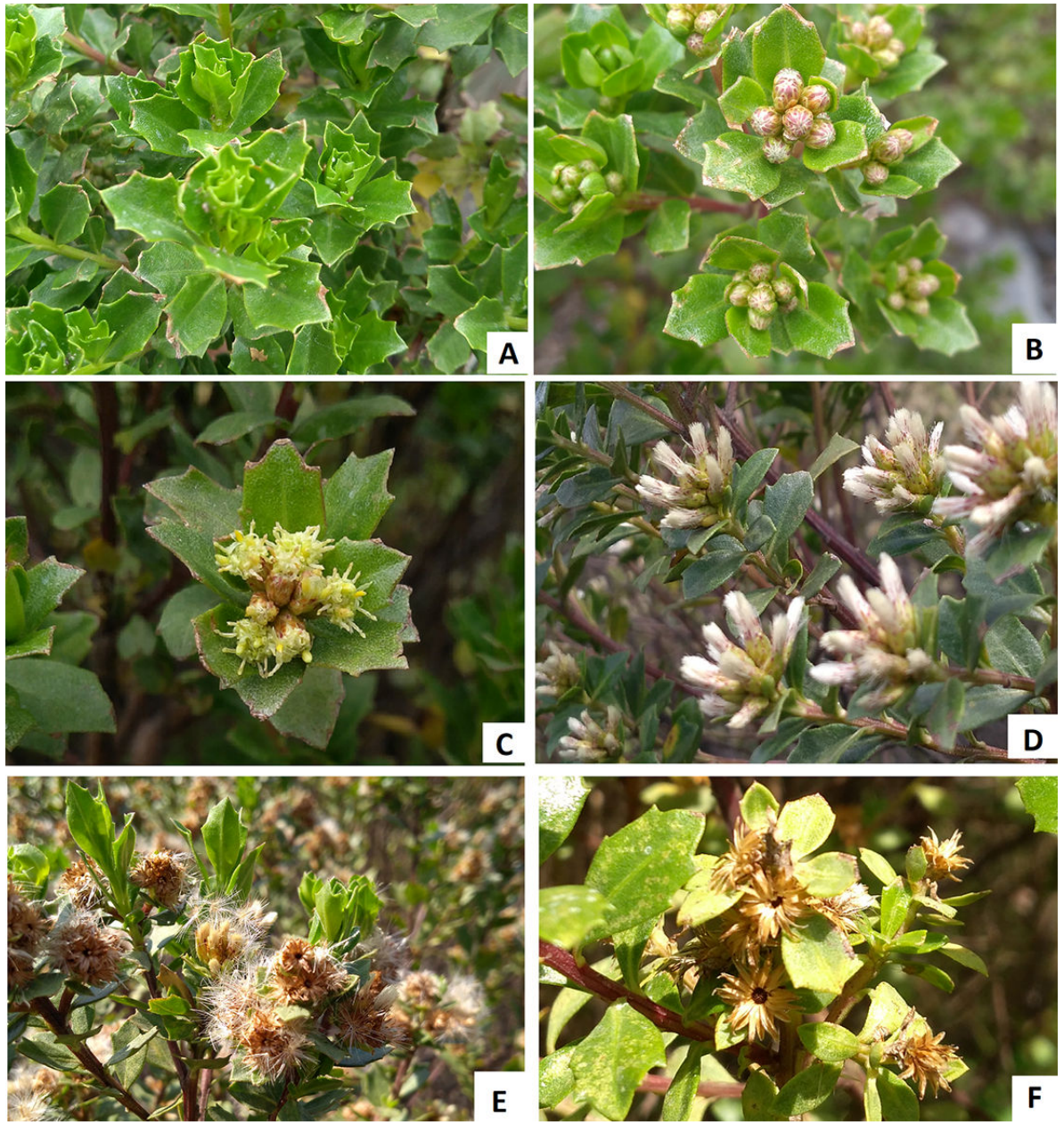

Figura 1. Fenofases de Baccharis conferta en el Parque Nacional Cofre de Perote, Veracruz, México. A) producción de brotes, B) preantesis, C) antesis masculina, D) antesis femenina, E) producción de semillas y F) senescencia.

La cepa de Alternaria solani fue adquirida del acervo de la Colección de Microorganismos del Centro de Investigación y de Estudios Avanzados del Instituto Politécnico Nacional (CINVESTAV-IPN). Con un asa se tomó una muestra para reactivar la cepa de este hongo colocándola en una caja Petri con PDA, se incubó a $28^{\circ} \mathrm{C}$ hasta que el hongo presentara esporulación (20 días aprox.).

Una vez reactivada la cepa, se tomaron discos de agar de $0.8 \mathrm{~cm}$ de diámetro colocándolos en el centro de cajas Petri con PDA. Los tratamientos aplicados fueron: extractos etanólicos (concentraciones de 25 y $10 \mathrm{mg}$ ) provenientes de individuos crecidos a dos altitudes $(2,900$ y $3,450 \mathrm{~m})$, de dos fenofases (antesis y producción brotes) y diferente sexo (masculino y femenino), además se aplicó un testigo control positivo con $10 \mu \mathrm{g}$ de ingrediente activo de fungicida mancozeb (nombre comercial Mancozeb Micro $80 \mathrm{pH}$, VELSIMEX, S.A. de C.V.) y un testigo control negativo con $10 \mu \mathrm{L}$ de etanol al $96 \%$ (Tabla 3 ).

En este experimento, cada tratamiento se aplicó de manera individual en cajas Petri de $9 \mathrm{~cm}$ de diámetro, los tratamientos se colocaron con micropipeta directamente sobre el medio PDA a una distancia de $2 \mathrm{~cm}$ del hongo. Para cada tratamiento se realizaron tres repeticiones. El cultivo se sometió a incubación por 7 días a $28{ }^{\circ} \mathrm{C}$ en una incubadora marca Binder. La variable de respuesta fue la inhibición de crecimiento del hongo (la distancia entre el crecimiento micelial y los tratamientos o en su caso, la invasión o no del micelio sobre los tratamientos).

Análisis de datos. Con los datos fenológicos se realizaron dos tablas de contingencia para responder a las preguntas 1 y 2 (¿Son diferentes las fenofases vegetativas y reproductivas de $B$. conferta entre organismos de diferente sexo? y ¿Son diferentes las fenofases vegetativas y reproductivas de Baccharis conferta a lo largo de un gradiente altitudinal?). La primera tabla fue de tamaño $(12 \times 5) \times 2$, la segunda $(12 \times 5) \times 3$, haciendo referencia a los 12 meses del año, cinco fenofases, dos sexos y tres altitudes. Los análisis se hicieron a través de una prueba de $\chi^{2}$ usando la función assoc del paquete "vcd" (Meyer et al. 


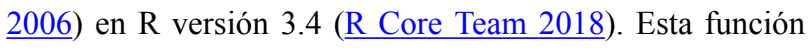
realiza gráficas de relación extendidas que indican las desviaciones de un modelo de independencia específico en una tabla de contingencia.

Tabla 2. Tratamientos aplicados en el experimento de Baccharis conferta sobre Myrothecium roridum in vitro. Del 1 al 35 representa el número de tratamiento.

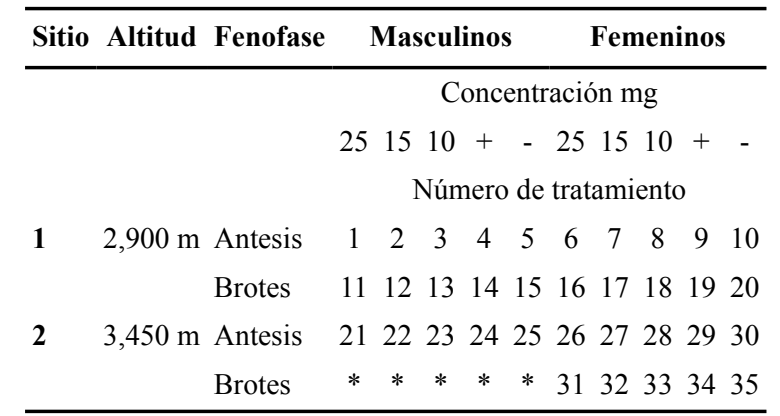

*Tratamientos no incluidos en M. roridum debido a que no se obtuvo extracto suficiente para el experimento. + Tratamiento control positivo (carbendazim), - Tratamiento control negativo (etanol).

El efecto de los extractos de B. conferta sobre M. roridum, se midió después de 7 días de incubación. A diferencia de otros trabajos donde consideran el crecimiento micelial (López-Mora 2013), nosotros consideramos la distancia entre el micelio y el tratamiento como variable de respuesta; a mayor distancia mayor el efecto. En el modelo incluimos como variables independientes la concentración de los tratamientos, el sexo, la fenofase, la altitud y sus interacciones. La variable respuesta fue la distancia $(\mathrm{mm})$ transformada en arcoseno de su raíz cuadrada (Zar 2010). Las comparaciones múltiples se realizaron con el método de ajuste de Bonferroni. Para los análisis se utilizó un modelo lineal generalizado (GLM por sus siglas en inglés) de SAS (2003).

Tabla 3. Tratamientos aplicados en el experimento de Baccharis conferta sobre Alternaria solani in vitro. Del 1 al 32 representa el número de tratamiento.

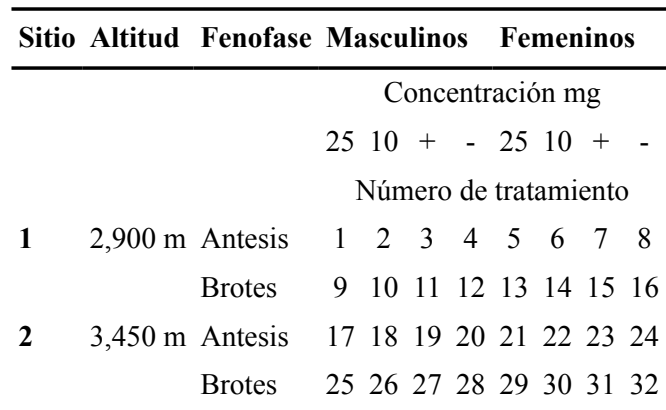

+ Tratamiento control positivo (mancozeb), - Tratamiento control negativo (etanol).
Para el caso de A. solani, el efecto de los extractos se midió a través de la invasión o no del micelio sobre los tratamientos (presencia-ausencia del micelio, en este caso el micelio del hongo creció más cerca de los tratamientos). Las variables independientes fueron las concentraciones de los tratamientos, el sexo, la fenofase, la altitud y sus posibles interacciones y la variable respuesta la invasión o no del micelio sobre el tratamiento. Los modelos fueron analizados a través de un modelo linela generalizado (GENMOD) de SAS, con distribución binomial.

\section{Resultados}

Fenología. La fenología vegetativa en el sitio uno (2,900 $\mathrm{m} \mathrm{snm})$ ocurrió de julio a diciembre (seis meses), en el sitio dos (3,450 m snm) de septiembre a noviembre (tres meses) y en el sitio tres $(3,670 \mathrm{~m} \mathrm{snm})$ únicamente en septiembre, registrando una fenología vegetativa de mayor duración en la menor altitud. Mientras que la fenología reproductiva en el sitio uno fue de enero a junio (seis meses), en el sitio dos inició en diciembre y terminó en agosto (nueve meses) y en el sitio tres persistió desde octubre hasta agosto del siguiente año (11 meses). Por lo que a mayor altitud se registró una fenología reproductiva más prolongada (Tabla 4). Cabe señalar que todos los individuos fenológicamente estaban sincronizados dentro de los sexos, tiempos y altitudes.

En el mes de septiembre, todos los individuos de todos los sitios se sincronizaron con la producción de brotes (Tabla 4). Asimismo, con excepción de los organismos ubicados a 3,670 m snm, la preantesis se observó de diciembre a febrero y los tres sitios mostraron antesis en primavera (Tabla 4). Sin embargo, el periodo más prolongado de antesis tuvo lugar en el sitio tres (ubicado a mayor altitud), persistiendo por siete meses (Tabla 4). La producción de semillas se registró en el mes de abril a 2,900 m snm y en junio-julio a 3,450 m y $3,670 \mathrm{~m}$ snm. Mientras que la senescencia de las partes reproductivas se observó en periodos más reducidos en organismos femeninos; en mayo y junio a 2,900 $\mathrm{m}$ y en agosto a $3,450 \mathrm{y}$ $3,670 \mathrm{~m} \mathrm{snm}$ (Tabla 4).

No encontramos una interacción significativa entre el sexo, la altitud y las fenofases $\chi^{2}=25.43, p=0.28$. Pero con relación a la primera pregunta de investigación ¿son diferentes las fenofases vegetativas y reproductivas de $B$. conferta entre organismos de diferente sexo? Los resultados señalan diferencias en las fenofases de producción de semillas y senescencia a nivel de sexo. Esto debido a que la producción de semillas únicamente se presenta en organismos femeninos y se determinó una asociación positiva entre la senescencia y los organismos masculinos en el mes de junio $\left(\chi^{2}=146.95, p=0.002\right.$; Figura 2). Los resultados también mostraron asociaciones 
Tabla 4. Comparación fenológica de Baccharis conferta por sexo en tres sitios ubicados a diferente altitud en el Parque Nacional Cofre de Perote, Veracruz, México.

\begin{tabular}{|c|c|c|c|c|c|c|}
\hline \multirow[t]{2}{*}{ Fenofase } & \multicolumn{3}{|c|}{$\begin{array}{c}\text { Masculinos } \\
\text { Altitud m snm }\end{array}$} & \multicolumn{3}{|c|}{$\begin{array}{c}\text { Femeninos } \\
\text { Altitud m snm }\end{array}$} \\
\hline & 2,900 & 3,450 & 3,670 & 2,900 & 3,450 & 3,670 \\
\hline $\begin{array}{l}\text { Producción de } \\
\text { brotes }\end{array}$ & $\begin{array}{c}\text { Julio, agosto, } \\
\text { septiembre, } \\
\text { octubre, noviembre } \\
\text { y diciembre }\end{array}$ & $\begin{array}{l}\text { Septiembre, octubre } \\
\text { y noviembre }\end{array}$ & Septiembre & $\begin{array}{l}\text { Julio, agosto, } \\
\text { septiembre, } \\
\text { octubre, } \\
\text { noviembre y } \\
\text { diciembre }\end{array}$ & $\begin{array}{c}\text { Septiembre, } \\
\text { octubre y } \\
\text { noviembre }\end{array}$ & Septiembre \\
\hline Preantesis & Enero y febrero & Diciembre y enero & Octubre & Enero y febrero & Diciembre y enero & Octubre \\
\hline Antesis & Marzo & $\begin{array}{l}\text { Febrero, marzo, } \\
\text { abril y mayo }\end{array}$ & $\begin{array}{l}\text { Noviembre, } \\
\text { diciembre enero, } \\
\text { febrero marzo, } \\
\text { abril y mayo }\end{array}$ & Marzo & $\begin{array}{c}\text { Febrero, marzo, } \\
\text { abril y mayo }\end{array}$ & $\begin{array}{c}\text { Noviembre } \\
\text { diciembre, enero, } \\
\text { febrero, marzo, } \\
\text { abril y mayo }\end{array}$ \\
\hline $\begin{array}{l}\text { Producción de } \\
\text { semillas }\end{array}$ & NA & NA & NA & Abril & Junio y julio, & Junio y julio \\
\hline Senescencia & $\begin{array}{l}\text { Abril, mayo y } \\
\text { junio }\end{array}$ & Junio, julio y agosto & $\begin{array}{c}\text { Junio, julio y } \\
\text { agosto }\end{array}$ & Mayo y junio & Agosto & Agosto \\
\hline
\end{tabular}

NA: No aplica por que los organismos masculinos no producen de semillas.

positivas entre las fenofases producción de brotes, preantesis y antesis en ambos sexos con los meses de septiembre, enero y marzo, respectivamente $\left(\chi^{2}=146.95\right.$, $p=0.002$; Figura 2).

Con relación a la segunda pregunta ¿son diferentes las fenofases vegetativas y reproductivas de Baccharis conferta a lo largo de un gradiente altitudinal? Los resultados mostraron una asociación positiva entre la producción de brotes y la altitud de 2,900 m, una asociación negativa entre la antesis y la altitud de 2,900 m y una asociación positiva entre la antesis y la altitud de $3,670 \mathrm{~m}\left(\chi^{2}=17.954\right.$, $p=0.021$ en todos los casos; Figura 3 ).

Bioprospección in vitro de extractos etanólicos relacionados con la fenología, altitud y sexo de plantas. Efecto de Baccharis conferta sobre Myrothecium roridum. La inhibición de los tratamientos con extractos etanólicos de $B$. conferta sobre el crecimiento micelial de $M$. roridum, fueron significativamente diferentes $(F=83, p<0.001)$, de la misma manera lo fueron entre concentraciones $(F=303$, $p<0.001)$, sexo $(F=21, p<0.001)$, fenofases (brotes y antesis) $(F=8, p=0.005)$ y altitudes $(F=15, p=0.002)$. Asimismo, fueron diferentes las interacciones concentraciones con sexo $(F=9.3, p<0.001)$, concentraciones con fenofases $(F=3.82, p=0.005) \mathrm{y}$ fenofase con altitud $(F=29, p<0.001$; Tablas 5 , $\underline{6}$ y $\underline{7})$.

La inhibición del crecimiento de $M$. roridum fue significativamente mayor con la interacción de la concentración de extracto de $10 \mathrm{mg}$ y el sexo femenino de $B$. conferta, sin embargo, presentó menor inhibición que el tratamiento positivo (carbendazim; Tabla 5). De igual manera, la interacción concentración de extracto etanólico de $10 \mathrm{mg}$ y la fenofase antesis tuvo mayor inhibición que el resto de las interacciones, los controles positivos presentaron mayor inhibición en comparación a todos los tratamientos con extractos (Tabla 6). Por último, las interacciones: antesis a 2,900 $\mathrm{m}$ snm y producción de brotes a 3,450 $\mathrm{m}$ snm presentaron inhibición del crecimiento sobre M. roridum ( $p<0.05$ en ambos casos; Tabla 7).

En la figura 4 se observa la diferencia del efecto de los tratamientos; donde el tratamiento realizado con organismos femeninos con brotes $(3,450 \mathrm{~m}$ snm $)$ y organismos femeninos en antesis $(2,900 \mathrm{~m} \mathrm{snm})$ presentaron mayor inhibición (Figura $4 \mathrm{C} \mathrm{y} \mathrm{G}$, respectivamente).

Efecto de Baccharis conferta sobre Alternaria solani. Los controles que resultaron positivo y/o negativo no se usaron en el modelo debido a que en todos los casos fueron 0 y 1 , respectivamente $(\mathrm{n}=6)$. El mejor modelo lineal generalizado fue el que incluyó: el sexo $\left(\chi^{2}=11.22\right.$, $p<0.001)$, las fenofases $\left(\chi^{2}=42.99, p<0.001\right)$ y las altitudes $\left(\chi^{2}=4.89, p=0.027\right)$ con diferencias significativas. Los tratamientos con mayor inhibición sobre A. solani fueron extractos femeninos y masculinos, en antesis y brotes, pero sólo a la altitud de 3,450 m snm (Tabla 8). 


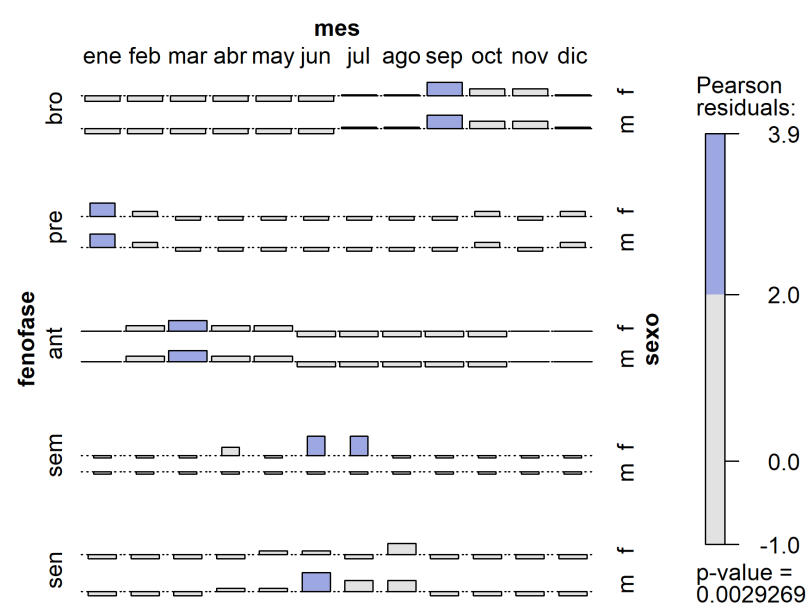

Figura 2. Asociaciones de Baccharis conferta entre sus fases fenológicas (bro: brotes, pre: preantesis, ant: antesis, sem: semillas y sen: senescencia), el sexo (f: femenino y m: masculino) y los meses del año. El color azul intenso representa las asociaciones positivas significativas.

\section{Discusión}

Fenología. Se muestra una amplia variación en la duración de las fenofases de $B$. conferta en las diferentes altitudes y sexos. Por ejemplo, la producción de brotes ocurre durante varios meses, pero es significativamente mayor en la altitud más baja (Figura 3). Esto sugiere que, a mayores altitudes (es decir a menor temperatura) se desencadena un cambio significativo en las fenofases de $B$. conferta. Esta respuesta, coincide con los resultados observados para otras especies, por ejemplo, en el haya europea (Fagus sylvatica L.) y el roble (Quercus robur L.), se observó que los cambios en el clima afectan su respuesta fenológica (Čufar et al. 2012, Dittmar \& Elling 2006, Guyon et al. 2010).

La producción de brotes ocurrió en septiembre en todos los sitios (Tabla 4), esto coincide con que este mes exhibe la mayor precipitación del año (Climate-data.org 2020). Lo anterior contrasta con otros estudios realizados en especies de sotobosque del bosque de Abies religiosa las cuales reportan una relación significativa, a mayor precipitación mayor floración (Bonilla-Valencia et al. 2017). Con excepción de los organismos ubicados a 3,670 m snm, la preantesis se observó en enero y febrero, y se encontró una relación positiva entre la preantesis y el mes de enero (Figura 2), siendo el mes con menor precipitación en esta región (Climate-data.org 2020). La floración y fructificación que observamos se presentó de noviembre a julio (nueve meses) a 3,670 $\mathrm{m}$ snm, estos resultados muestran un periodo más amplio de dichas fenofases con relación a los reportados anteriormente por Vázquez (2014) el cual fue de enero a junio a 3,000, a 3,600 y a 4,000 m snm, así como los resultados de Cornejo-Tenorio \& IbarraManríquez (2007) quienes las reportan de enero a julio en altitudes de 2,500 a $3,320 \mathrm{~m}$ snm. La producción de semillas se relacionó positivamente con los meses de junio y julio (Figura 2), en contraste con la producción de semillas en $B$. dracunculifolia que ocurre en la estación seca de Belo Horizonte, Brasil (Espírito-Santo 2003).

Finalmente podemos concluir que la fenología de $B$. conferta es diferente a través de un gradiente altitudinal y se asocia inversamente con la temperatura, principalmente, es decir a mayor altitud menor temperatura. El principal factor climático que afecta la fenología de las plantas es la temperatura (Menzel 2000), el fotoperiodo y la precipitación (Donohue et al. 2015), esto sugiere que estos son los principales factores que causan la variación en la fenología de $B$. conferta a lo largo del gradiente altitudinal. Es también conocido que la fenología está regulada por la expresión genética del Flowering Locus C (FLC represor de la floración sensible a la temperatura y al fotoperiodo), un FLC homólogo o un locus equivalente v.gr., Oryza sativa L., Populus trichocarpa Torr. \& A. Gray y Fagus crenata Blume y el trigo, respectivamente (Kudoh 2016).

Aunque el tipo de suelo en los tres sitios es Andosol, puede haber variaciones en su contenido de nutrientes y $\mathrm{pH}$, esto también podría contribuir a la variación de la fenología de las plantas. Nosotros sostenemos la hipótesis de que la temperatura y la precipitación son los factores que más contribuyen a la variación de la fenología. Sin embargo, se deberá probar en otro estudio donde se incluyan los factores suelo - individuo - sexo - altitud - fotoperiodo precipitación - genes junto son sus interacciones para evaluar la contribución de cada uno de estos factores sobre la variación de la fenología de $B$. conferta.

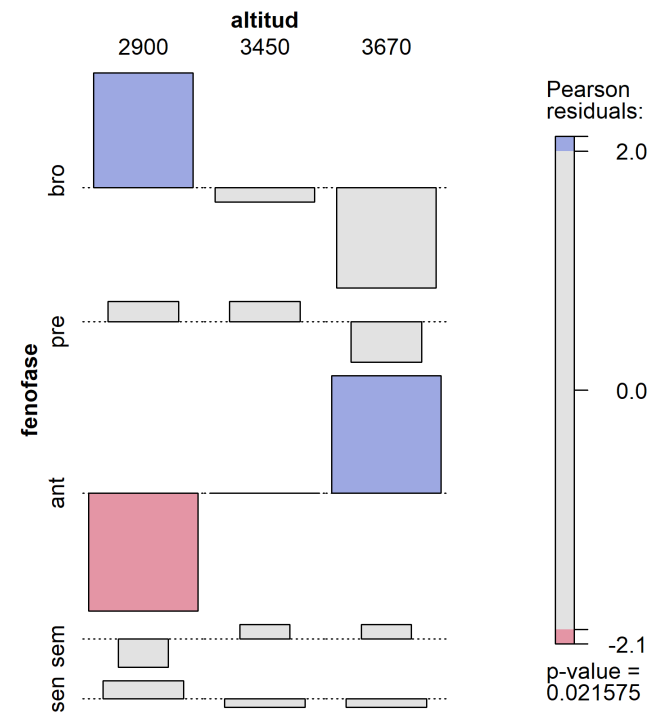

Figura 3. Asociaciones entre la altitud y las fases fenológicas de Baccharis conferta (bro: brotes, pre: preantesis, ant: antesis, sem: semillas y sen: senescencia), el color azul intenso representa asociaciones positivas significativas y el color rosa asociaciones negativas significativas. 
Tabla 5. Efecto de los tratamientos concentración extracto y sexo de Baccharis conferta sobre la inhibición del crecimiento (distancia) de Myrothecium roridum in vitro. LSMean mínimos cuadrados promedio, por sus siglas en inglés.

\begin{tabular}{|c|c|c|c|c|}
\hline Concentración del extracto & Sexo & $\begin{array}{l}\text { LSMean } \\
\text { Inhibición }\end{array}$ & $\begin{array}{l}\text { Número de la } \\
\text { interacción }\end{array}$ & $\begin{array}{l}\text { Diferencias significativas }(p<0.05) \text { entre } \\
\text { interacción concentración y sexo }\end{array}$ \\
\hline $10 \mathrm{mg}$ & Femenino & 0.16666667 & 1 & 1 vs $2,3,4,5,6,7,8$ \\
\hline $10 \mathrm{mg}$ & Masculino & -0.00511111 & 2 & 2 vs 1,8 \\
\hline $15 \mathrm{mg}$ & Femenino & 0.02500000 & 3 & 3 vs 1,8 \\
\hline $15 \mathrm{mg}$ & Masculino & 0.00322222 & 4 & 4 vs 1,8 \\
\hline $25 \mathrm{mg}$ & Femenino & 0.02500000 & 5 & 5 vs 1,8 \\
\hline $25 \mathrm{mg}$ & Masculino & 0.00322222 & 6 & 6 vs 1,8 \\
\hline $\begin{array}{l}\text { Control negativo } 10 \mu \mathrm{L} \text { de etanol } \\
(96 \%)\end{array}$ & Control & 0.00322222 & 7 & 7 vs 1,8 \\
\hline $\begin{array}{l}\text { Control positivo } 0.0125 \mu \mathrm{g} \\
\text { carbendazim }\end{array}$ & Control & 0.65655556 & 8 & 8 vs $1,2,3,4,5,6,7$ \\
\hline
\end{tabular}

Relación entre fenología, altitud y sexo con la bioprospección in vitro. Efecto de Baccharis conferta sobre Myrotecium roridum. El ambiente (altitud) donde crece B. conferta, sus fenofases, las concentraciones de extractos y el tipo de sexo son factores que interactúan y su efecto varía sobre el crecimiento (inhibición) de $M$. roridum (Tablas $5, \underline{6}$ y 7 ). Es decir, su efecto se complejiza con las posibles interacciones entre los niveles de estos factores. Además, se observó que no necesariamente son las mayores concentraciones de los tratamientos los que tienen mejores efectos cuando hay otros factores con los que interactúan, por ejemplo, el tratamiento a una concentración de $10 \mathrm{mg}$ con plantas femeninas en la fenofase antesis, presentó mayor actividad respecto a otros tratamientos con mayor concentración (Tablas 6 y 7 ).
Los resultados sugieren que la concentración de $10 \mathrm{mg}$ de extractos de plantas de sexo femenino, así como la interacción de antesis con la altitud 2,900 $\mathrm{m}$ y los brotes a $3,450 \mathrm{~m} \mathrm{snm}$, son las mejores combinaciones para la búsqueda de metabolitos secundarios con actividad antifúngica en $B$. conferta. Cabe mencionar que el periodo de colecta de material vegetal se puede reducir ya que hay fenofases como la floración que se reducen a un mes del año (marzo), en el sitio de menor altitud.

Efecto de Baccharis conferta sobre Alternaria solani. El uso de extractos etanólicos de $B$. conferta y su efecto sobre el crecimiento in vitro de $A$. solani, también nos permitió identificar lo importante de considerar en las colectas de plantas el sexo, las fenofases y altitudes. Por ejemplo, los

Tabla 6. Resultados del efecto de las interacciones, concentraciones de extractos etanólicos de Baccharis conferta y la fenofase de las plantas, sobre la inhibición (distancia) del crecimiento de Myrothecium roridum in vitro. LSMean es mínimos cuadrados promedio, por sus siglas en inglés.

\begin{tabular}{|c|c|c|c|c|}
\hline Concentración del extracto & Fenofase & $\begin{array}{l}\text { LSMean } \\
\text { Inhibición }\end{array}$ & $\begin{array}{l}\text { Número de la } \\
\text { interacción }\end{array}$ & $\begin{array}{l}\text { Diferencias significativas }(p<0.05) \text { entre } \\
\text { interacción concentración y fenofase }\end{array}$ \\
\hline $10 \mathrm{mg}$ & Antesis & 0.11666667 & 1 & 1 vs $3,4,5,6,7,8$ \\
\hline $10 \mathrm{mg}$ & Brotes & 0.04488889 & 2 & 2 vs 8 \\
\hline $15 \mathrm{mg}$ & Antesis & 0.02500000 & 3 & 3 vs 1,8 \\
\hline $15 \mathrm{mg}$ & Brotes & 0.00322222 & 4 & 4 vs 1,8 \\
\hline $25 \mathrm{mg}$ & Antesis & 0.02500000 & 5 & 5 vs 1,8 \\
\hline $25 \mathrm{mg}$ & Brotes & 0.00322222 & 6 & 6 vs 1,8 \\
\hline $\begin{array}{l}\text { Control negativo } 10 \mu \mathrm{L} \text { de etanol } \\
(96 \%)\end{array}$ & Control & 0.00322222 & 7 & 7 vs 1,8 \\
\hline $\begin{array}{l}\text { Control positivo } 0.0125 \mu \mathrm{g} \\
\text { carbendazim }\end{array}$ & Control & 0.66488889 & 8 & 8 vs $1,2,3,4,5,6,7$ \\
\hline
\end{tabular}


Bioprospección modulada por la fenología, el sexo y el gradiente altitudinal

Tabla 7. Resultados del efecto de las interacciones, fenofase y la altitud de las plantas sobre la inhibición (distancia) del crecimiento de Myrothecium roridum in vitro. LSMean es mínimos cuadrados promedio, por sus siglas en inglés.

\begin{tabular}{lcccc}
\hline Fenofase & Altitud & $\begin{array}{c}\text { LSMEAN } \\
\text { Inhibición }\end{array}$ & $\begin{array}{c}\text { Número de la } \\
\text { interacción }\end{array}$ & $\begin{array}{c}\text { Diferencias significativas }(\boldsymbol{p}<\mathbf{0 . 0 5}) \text { entre } \\
\text { interacción fenofase y altitud }\end{array}$ \\
\hline $\begin{array}{l}\text { Control negativo } 10 \mu \mathrm{L} \mathrm{de} \\
\text { etanol }(96 \%)\end{array}$ & Control & 0.02500000 & 1 & 1 vs 2,3 \\
$\begin{array}{l}\text { Control positivo } 0.0125 \mu \mathrm{g} \\
\text { carbendazim }\end{array}$ & Control & 0.60833333 & 2 & 2 vs $1,3,4,5,6$ \\
Antesis & 2,900 & 0.10555556 & 3 & 3 vs $1,2,4,5$ \\
Antesis & 3,450 & 0.00555556 & 4 & 4, vs 2,3 \\
Brotes & 2,900 & 0.00000001 & 5 & 5 vs 2,3 \\
Brotes & 3,450 & 0.06666667 & 6 & 6 vs 2 \\
\hline
\end{tabular}

mejores resultados se obtuvieron con individuos femeninos y masculinos, en ambas fenofases, pero únicamente en la altitud de $3,450 \mathrm{~m} \mathrm{snm}$ (Tabla 8); y a diferencia con M. roridum, la concentración de $25 \mathrm{mg}$ obtuvo el mejor resultado.

Comparando los resultados y tendencias. Los experimentos realizados en este trabajo sugieren claramente que en los estudios de bioprospección en $B$. conferta, los metabolitos secundarios en extractos etanólicos difieren de acuerdo con la procedencia altitudinal, fenológica y sexo de la planta. En B. dracunculifolia también se ha demostrado la variación de metabolitos secundarios antifúngicos en hojas ( $\mathrm{Da}$ Silva 2008). Sin embargo, no podemos comparar nuestros resultados debido a que en ese estudio no se especifica el sexo y la altitud donde fue colectada. Este dato es importante ya que se han comparado los perfiles cromatográficos de las hojas maduras y en brotes de B. dracunculifolia y observaron que las hojas maduras no contenían o tenían muy reducidos los metabolitos secundarios de interés, a diferencia de los brotes (Park et al. 2004). La actividad antifúngica de los brotes puede explicarse también por la presencia de compuestos en altas concentraciones que las plantas presentan en tejidos periféricos (en su mayoría acoplados a un azúcar para prevenir la autotoxicidad), los cuales son los primeros en entrar en contacto con herbívoros y patógenos (Palo 1984).

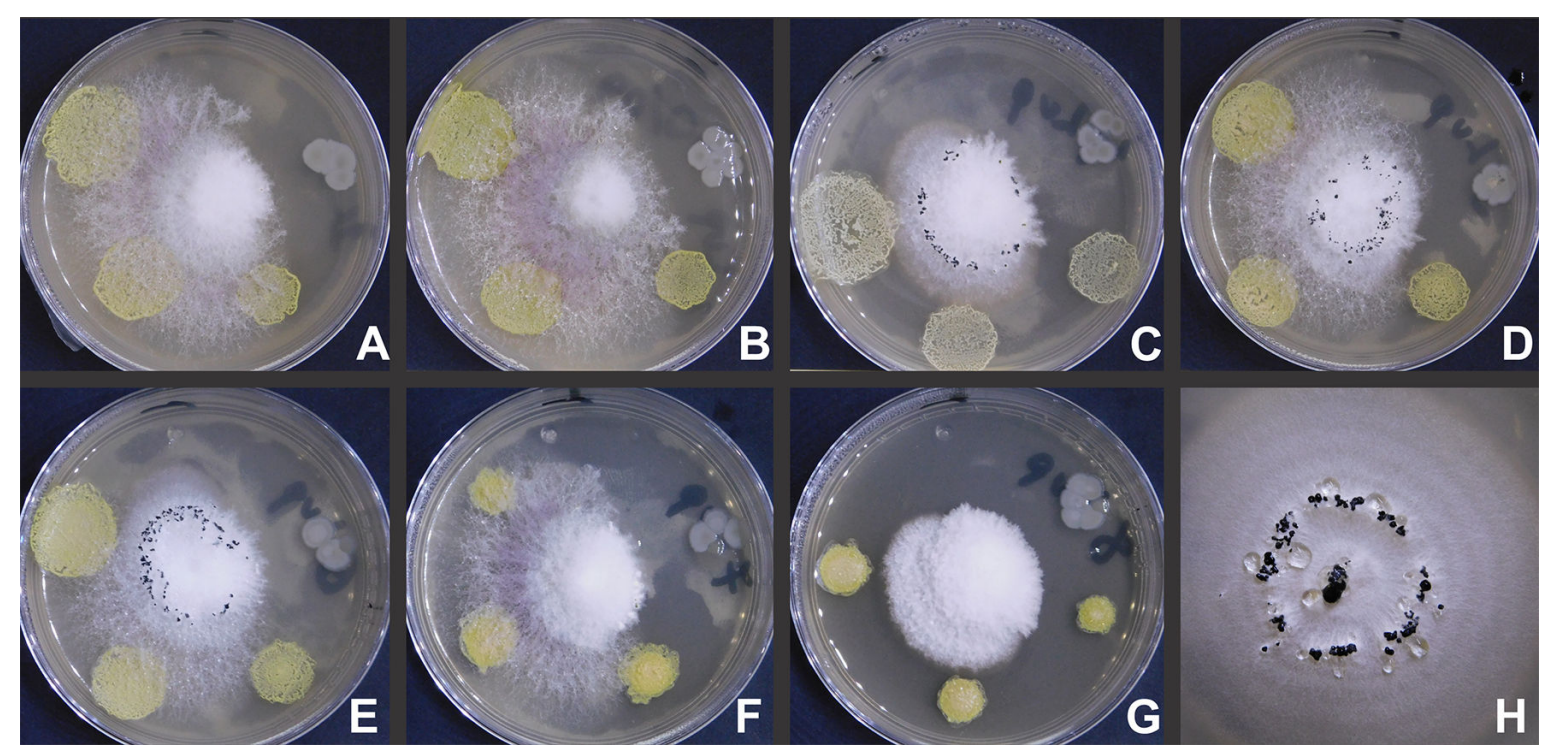

Figura 4. Efecto de Baccharis conferta sobre el crecimiento in vitro de Myrothecium roridum. Las letras indican los tratamientos: A) masculinos con brotes a 2,900 m snm, B) femeninos con brotes a 2,900 m snm, C) femeninos con brotes a 3,450 m snm, D) masculinos en antesis a 3,450 m snm, E) femeninos en antesis a 3,450 m snm, F) masculinos en antesis a 2,900 m snm, G) femeninos en antesis a 2,900 m snm, H) Control negativo. Nota: En todas las cajas (A-G) se puede observar en el lado derecho el control positivo carbendazim (gotas blancas), en todos esos sitios de las cajas no hubo crecimiento del hongo. 
Como lo señalan nuestros resultados, la bioprospección de especies dioicas debe hacerse por separado con ambos sexos. En Salix rigida Muhl. también se ha comparado el contenido de compuestos por sexo, determinando que los organismos femeninos presentan mayor concentración de metabolitos, así como metabolitos ausentes en organismos masculinos (Elmqvist et al. 1991), como resultado, los ratones de la especie Microtus agrestis L. prefieren alimentarse de los sauces (Salix myrsinifolia Salisb.) masculinos sobre los femeninos (Danell 1983).

Considerar la fenofase en la colecta del material vegetal para la bioprospección es relevante. En este estudio se observaron diferencias significativas entre fenofases y con interacción con los otros factores sobre M. roridum. Otro ejemplo similar se observó en Artemisia molinieri Quézel, M. Barbero \& R.J. Loisel, en la cual se han registrado variaciones en la composición de sus aceites esenciales en diferentes etapas fenológicas, específicamente en la fase vegetativa se reportó mayor cantidad de R-terpineno y tasas más bajas de ascaridol, mientras que, en la floración, la proporción de ascaridol aumentó mientras que la de Rterpineno disminuyó (Masotti et al. 2003). De igual manera, la concentración de metabolitos secundarios en Salix rigida se reduce en la fructificación (Elmqvist et al. 1991). En resumen, las fenofases pueden tener efectos diferenciados dependiendo del organismo al que se apliquen los extractos (Tablas 7 y $\underline{8}$ ).

Tabla 8. Respuesta de Alternaria solani a extractos etanólicos de Baccharis conferta. Modelo lineal generalizado binomial con variable respuesta (presencia-ausencia de la inhibición) y cuatro variables independientes.

\begin{tabular}{lcccc}
\hline Factor & Estimación & Error estándar & Valor $\boldsymbol{Z}$ & $\operatorname{Pr}>|\mathbf{z}|$ \\
\hline $\mathbf{1 0} \mathbf{~ m g}$ & 0.62878 & 0.8198 & 0.77 & 0.4438 \\
$\mathbf{2 5} \mathbf{~ m g}$ & 2.1098 & 1.1299 & 1.87 & 0.0619 \\
Femenino & 15.319 & 0.7438 & 20.59 & $<0.0001$ \\
Masculino & -12.579 & 1.3516 & -0.31 & $<0.0001$ \\
$\mathbf{2 , 9 0 0}$ m snm & $4.44 \mathrm{E}-16$ & 0.8234 & 0.00 & 1.0000 \\
$\mathbf{3 , 4 5 0}$ m snm & 2.7376 & 1.2071 & 2.27 & 0.0233 \\
Antesis & -14.4662 & 0.7306 & -19.80 & $<0.0001$ \\
Brotes & 17.20439 & 0.7306 & 23.55 & $<0.0001$ \\
\hline
\end{tabular}

La altitud (refiriéndonos a los factores ambientales asociados a ella), es otra de las variables que influyó en la actividad de los extractos sobre $M$. roridum y $A$. solani, en la primera solo con interacciones con la fenofase y en la segunda sin interacción con los otros factores (Tablas 7 y $\underline{8}$ ). Como antecedente, se ha reportado que el piso térmico donde se desarrollan las plantas puede influir en el contenido de polifenoles totales y taninos condensados en las especies Gliricidia sepium Kunth ex Steud. y Tithonia diversifolia (Hemsl.) A. Gray (Santacoloma-Varón \& Granados 2010) así como en la estructura y composición química de Abies religiosa (Musule et al. 2016).

Otro estudio, reporta la importancia de los factores ambientales sobre la concentración de metabolitos secundarios en Smilax campestris Griseb., en la cual las hojas más expuestas a la luz tienen mayor contenido de glicósidos de quercetina y canferol, así como con el doble de fenoles totales y niveles superiores de taninos. En este caso sólo se analizaron ejemplares femeninos para descartar que el sexo influía en los resultados (Rugna et al. 2007).

Todos los resultados mencionados incluyendo el nuestro, refuerzan la importancia de los estudios de bioprospección ligados a la fenología, el sexo y la altitud. Por lo tanto, es relevante considerar que las evaluaciones de actividad biológica o bioprospección se lleven a cabo incluyendo aquellos factores o por lo menos tomar nota sobre el sexo (en caso de ser dioico), el estado fenológico y la altitud a la cual se colecta el material botánico. Consideramos que esto debe formar parte de los conocimientos básicos sobre la utilización terapéutica de productos de origen vegetal, ya sean drogas, extractos, aceites esenciales u otros preparados utilizados también en la medicina tradicional, como parte de un control de calidad que garantice su eficacia. Incluso esta información debería compartirse con las comunidades que hacen uso de plantas medicinales, para que lleven a cabo un uso adecuado de las mismas.

Para finalizar, estos resultados también pueden ser de utilidad para los productores de plantas con uso medicinal para que consideren la altitud en la que preferentemente deben sembrar cada especie según los metabolitos secundarios que esperen obtener, así como la fenofase y el sexo en que deben ser colectados.

\section{Agradecimientos}

Agradecemos a Jorge Antonio Gómez Díaz, Rogelio Lara González, Eire Ramírez e Israel Estrada Contreras por su colaboración en campo. Los autores agradecen a los dos árbitros anónimos por sus comentarios y observaciones, los cuales enriquecieron y mejoraron la versión final del manuscrito. El primer autor agradece al CONACyT por la beca de doctorado número 285104. Este trabajo fue financiado por el proyecto "Caminando por la biodiversidad, la bioprospección, la ecología de poblaciones y comunidades, pernoctando en la ecología de la restauración" financiado por la Universidad Veracruzana, la familia Sánchez-Pineda y por el proyecto número 220206: "Búsqueda de metabolitos bioactivos en sistemas de arrecifes veracruzanos: estudios estructurales, farmacológicos y biotecnológicos", financiado por el CONACyT otorgado a F. A. Cen Pacheco. 


\section{Literatura citada}

Abad MJ, Bermejo P. 2007. Baccharis (Compositae): a review update. ARKIVOC 7: 76-96. DOI: $\underline{\text { http://dx.doi. }}$ org/10.3998/ark.5550190.0008.709

Angulo-Escalante MÁ, Armenta-Reyes E, García-Estrada RS, Carrillo-Fasio JA, Salazar-Villa E, \& Valdéz-Torres, JB. 2009. Extractos de Semilla de Swietenia humilis Zucc. con Actividad Antifúngica en Rhizopus stolonifer (Ehrenb.:Fr.) Vuill. Revista Mexicana de Fitopatología, 27: 84-92.

Bonilla-Valencia L, Martínez-Orea Y, Castillo-Argüero S, Barajas-Guzmán G, Romero-Romero M, Díaz-López E. 2017. Reproductive phenology of understory species in an Abies religiosa (Pinaceae) forest in the Magdalena River Basin, Mexico City. The Journal of the Torrey Botanical Society 144: 313-327. DOI: https://doi.org/ 10.3159/TORREY-D-16-00024.1

Bunster L, Torres M. 2003. Incidencia de Myrothecium roridum Tode: Fries como agente de la pudrición del cuello de plantas de pensamiento (Viola tricolor L.) en la provincia de Quillota, Chile. Boletín Micológico 18: 39-45. DOI: https://doi.org/10.22370/bolmicol.2003.18. $\underline{0.406}$

Cabral CS, Henz GP, Moreira AJA, Reis A. 2009. New cucurbitaceous hosts of Myrothecium roridum in Amazonas State, Brazil. Tropical Plant Pathology 34: 402-405. DOI: https://doi.org/10.1590/S1982-56762009 $\underline{000600006}$

Calderón de Rzedowski G, Rzedowski J. 2005. Flora fanerogámica del Valle de México. Pátzcuaro, Michoacán: Instituto de Ecología A.C. - Comisión Nacional para el Conocimiento y Uso de la Biodiversidad. ISBN: 978-607-7607-36-6

Carvajal-Hernández C, Krömer T. 2015. Riqueza y distribución de helechos y licófitos en el gradiente altitudinal del Cofre de Perote, centro de Veracruz, México. Botanical Sciences 93: 601-614. DOI: http:// dx.doi.org/10.17129/botsci.165

Graves C. 1996. La papa tesoro de los Andes, De la agricultura a la cultura. Lima, Perú: Centro Internacional de la Papa. ISBN: 92-9060-188-4

Climate-data.org. 2020. https://es.climate-data.org/americadel-norte/mexico/veracruz-de-ignacio-de-la-llave/perote46140/ (accessed January 4, 2020).

Conrado M, Da Silva J, Maia GL, Bueno C, De Souza N. 2006. Mancha de mirotécio em algodoeiro causada por Myrothecium roridum. Summa Phytopathologica Botucatu 32: 390-393. DOI: http://dx.doi.org/10.1590/ $\underline{\text { S0100-54052006000400016 }}$

Cornejo-Tenorio G, Ibarra-Manríquez G. 2007. Plant reproductive phenology in a temperate forest of The Monarch Butterfly Biosphere Reserve, Mexico.
Interciencia 32: 445-452. DOI: https://www.redalyc.org/ articulo.oa?id $=33932704$

Čufar K, De Luis M, Saz M, Črepinšek Z, Kajfež-Bogataj L. 2012. Temporal shifts in leaf phenology of beech (Fagus sylvatica) depend on elevation. Trees 26: 1091-1100. DOI: https://doi.org/10.1007/s00468-012-06 $\underline{86-7}$

Danell K, Elmqvist T, Ericson L, Salomonson A. 1983. Sexuality in Willows and Preference by Bark-Eating Voles: Defence or Not? Oikos 44: 82-90. DOI: https:// doi.org/10.2307/3544047

Da Silva A, De Sousa J, Soares S, Furtado N, Andrade M, Cunha W, Gregório L, Dhammika N, Bastos J. 2008. Antimicrobial Activity of the Extract and Isolated Compounds from Baccharis dracunculifolia D. C. (Asteraceae). Zeitschrift für Naturforschung C 63: 40-46. DOI: https://doi.org/10.1515/znc-2008-1-208

Da Silveira SF, Dias VM, De Ponte EC, Dias PP. 2007. Mancha de Mirotécio em Mudas de Cafeeiro. Fitopatologia Brasileira 32: 5. DOI: http://dx.doi.org/ 10.1590/S0100-41582007000500013

Dittmar C, Elling W. 2006. Phenological phases of common beech (Fagus sylvatica L.) and their dependence on region and altitude in Southern Germany. European Journal of Forest Research 125: 181-188. DOI: https:// doi.org/10.1007/s10342-005-0099-x

Donohue K, Burghardt LT, Runcie D, Bradford KJ, Schmitt J. 2015. Applying developmental threshold models to evolutionary ecology. Trends in Ecology \& Evolution 30: 66-77. DOI: https://doi.org/10.1016/j.tree.2014.11.008

Elmqvist T, Cates R, Harper J, Gardfjell H. 1991. Flowering in males and females of a Utah willow, Salix rigida and effects on growth, tannins, phenolic glycosides and sugars. Oikos 61: 65-72. DOI: https:// www.jstor.org/stable/3545407

Espírito-Santo M, Madeira B, Neves F, Faria M, Fagundes M, Fernades G. 2003. Sexual differences in reproductive phenology and their consequences for the demography of Baccharis dracunculifolia (Asteraceae), a dioecious tropical shrub. Annals of Botany 91: 13-19. DOI: https:// doi.org/10.1093/aob/mcg001

Feresin GE, Tapia AA, Bustos BA. 2000. Antibacterial activity of some medicinal plants from San Juan, Argentina. Fitoterapia 71: 429-432. DOI: https://doi.org/ 10.1016/S0367-326X(00)00128-3

Fernando T, Jarvis B, Bean G. 1986. A comparison of the effect of three fungicides on growth and roridin $\mathrm{E}$ production by Myrothecium roridum. Mycopathologia 95: 155-158. DOI: https://doi.org/10.1007/BF00437120

Fish WW, Bruton BD, Popham TW. 2012. Cucurbit host range of Myrothecium roridum isolated from watermelon. American Journal of Plant Sciences 3: 353-359. DOI: http://dx.doi.org/10.4236/ajps.2012.33 $\underline{042}$ 
Flores-Estévez N, Vasquez-Morales SG, Cano-Medina T, Sánchez-Velásquez LR, Noa-Carrazana JC, DíazFleischer F. 2013. Insecticidal activity of raw ethanolic extracts from Magnolia dealbata Zucc on a tephritid pest. Journal of Environmental Science and Health, Part $B$ 48: 582-586. DOI: https://doi.org/10.1080/03601234. $\underline{2013.774933}$

Funes M, Garro M, Tosso R, María A, Saad J, Enriz R. 2018. Antinociceptive effect of neo-clerodane diterpenes obtained from Baccharis flabellata. Fitoterapia 130: 94-99. DOI: https://doi.org/10.1016/j.fitote.2018.08.017

Guyon D, Guillot M, Vitasse Y, Cardot H, Hagolle O, Delzon S, Wigneron J. 2010. Monitoring elevation variations in leaf phenology of deciduous broadleaf forests from SPOT/VEGETATION time-series. Remote Sensing of Environment 115: 615-627. DOI: https://doi. org/10.1016/j.rse.2010.10.006

Haudenshield JS, Pawlowski M, Miranda C, Hartman GL. 2018. First report of Paramyrothecium roridum causing Myrothecium leaf spot on soybean in Africa. Plant Disease 102: 2638. DOI: https://doi.org/10.1094/PDIS -04-18-0624-PDN

Kudoh H. 2016. Molecular phenology in plants: in natura systems biology for the comprehensive understanding of seasonal responses under natural environments. New Phytologist 210: 399-412. DOI: https://doi.org/10.1111/ nph.13733

Kumar V, Haldar S, Pandey K, Singh R, Singh A, Singh P. 2008. Cultural, morphological, pathogenic and molecular variability amongst tomato isolates of Alternaria solani in India. World Journal of Microbiology and Biotechnology 24: 1003-1009. DOI: https://doi.org/ $\underline{\text { 10.1007/s11274-007-9568-3 }}$

Kurdelas R, Lima L, Tapia A, Egly-Feresin G, GonzalezSierra M, Rodríguez MV, Zacchino S, Enriz R, Freile M. 2010. Antifungal activity of extracts and prenylated coumarins isolated from Baccharis darwinii Hook \& Arn. (Asteraceae). Molecules 15: 4898-4907. DOI: https://doi.org/10.3390/molecules15074898

López-Mora LI, Gutiérrez-Martínez P, Bautista-Baños S, Jiménez-García LF, Zavaleta-Mancera HA. 2013. Evaluación de la actividad antifúngica del quitosano en Alternaria alternata y en la calidad del mango 'Tommy Atkins' durante el almacenamiento. Revista Chapingo Serie Horticultura 19: 315-331. DOI: http://dx.doi.org/ $\underline{\text { 10.5154/r.rchsh.2012.07.038 }}$

Marques A, Fernandes G, Reis I, Assunção R. 2008. Distribution of adult male and female Baccharis concinna (Asteraceae) in the rupestrian fields of Serra Do Cipó, Brazil. Plant Biology 4: 94-103. DOI: https:// doi.org/10.1055/s-2002-20441

Masotti V, Juteau F, Bessiere J, Viano J. 2003. Seasonal and phenological variations of the essential oil from the narrow endemic species Artemisia molinieri and its biological activities. Journal of Agricultural and Food Chemistry 51: 7115-712. DOI: https://doi.org/10.1021/ jf034621y

Menzel A. 2000. Trends in phenological phases in Europe between 1951 y 1996. International Journal of Biometeorology 44: 76-81. DOI: https://doi.org/10.1007/ $\underline{\mathrm{s} 004840000054}$

Meyer D, Zeileis A, Hornik K. 2006. The Strucplot Framework: Visualizing Multi-Way Contingency Tables with vcd.Journal of Statistical Software 17: 1-48. DOI: https://doi.org/10.18637/jss.v017.i03

Musule R, Alarcón-Gutiérrez E, Houbron EP, BárcenasPazos GM, Pineda-López MR, Domínguez Z, SánchezVelásquez LR. 2016. Chemical composition of lignocellulosic biomass in the wood of Abies religiosa across an altitudinal gradient. Journal of Wood Science 62: 537-547. DOI: https://doi.org/10.1007/s10086-016$1585-0$

Palo RT. 1984. Distribution of birch (Betula spp.), willow (Salix spp.), and poplar (Populus spp.) secondary metabolites and their potential role as chemical defense against herbivores. Journal of Chemical Ecology 10: 499-520. DOI: https://doi.org/10.1007/BF00988096

Park Y, Paredes-Guzmán J, Aguilar C, Alencar S, Fujiwara F. 2004. Chemical constituents in Baccharis dracunculifolia as the main botanical origin of southeastern brazilian propolis. Journal of Agricultural and Food Chemistry 52: 1100-110. DOI: https://doi.org/ 10.1021/jf021060m

Peraza-Sánchez SR, Cen-Pacheco F, Noh-Chimal A, MayPat F, Simá-Polanco P, Dumonteil E, Mut-Martín M. 2007. Leishmanicidal evaluation of extracts from native plants of the Yucatan peninsula. Fitoterapia 78: 315-318. DOI: https://doi.org/10.1016/j.fitote.2007.03.013

Rahalison L, Benathan M, Monod M, Frenk E, Gupta M, Solis P, Fuzzati N, Hostettmann K. 1995. Antifungal principles of Baccharis pedunculata. Planta Medica 61: 360-362. DOI: https://doi.org/10.1055/s-2006-958101

$\mathrm{R}$ Core Team. 2018. $R$, a language and environment for statistical computing. $R$ Foundation for Statistical Computing, Vienna. https://www.r-project.org/ (accessed November 1, 2019).

Rosas-Burgos E, Cortez-Rocha M, Cinco-Moroyoqui F, Robles-Zepeda R, López-Cervantes J, Sánchez-Machado D, Lares-Villa F. 2009. Antifungal activity in vitro of Baccharis glutinosa and Ambrosia confertiflora extracts on Aspergillus flavus, Aspergillus parasiticus and Fusarium verticillioides. World Journal of Microbiology and Biotechnology 25: 2257. DOI: https://doi.org/10.10 07/s11274-009-0116-1

Rugna A, Ricco R, Gurni A, Wagner M. 2007. Efectos de la radiación solar sobre la producción de polifenoles en 
ejemplares femeninos de Smilax Campestris Griseb. Smilacaceae. Latin America Journal of Pharmacy 26: 420-3.

Ruiz-Bustos E, Velazquez C, Garibay-Escobar A, García Z, Plascencia-Jatomea M, Cortez-Rocha MO, RoblesZepeda RE. 2009. Antibacterial and antifungal activities of some Mexican medicinal plants. Journal of Medicinal Food 12: 1398-1402. DOI: https://doi.org/10.1089/jmf. $\underline{2008.0205}$

Rzedowski J. 1978. Vegetación de México. México: LIMUSA. ISBN: 968-18-0002-8

Sánchez-Velásquez LR, Domínguez-Hernández D, PinedaLópez MR, Lara-González R. 2011. Does Baccharis conferta shrub act as a nurse plant to the Abies religiosa seedling? The Open Forest Science Journal 4: 67-70. DOI: https://doi.org/10.2174/1874398601104010067

Santacoloma-Varón LE, Granados J. 2010. Evaluación del contenido de metabolitos secundarios en dos especies de plantas forrajeras encontradas en dos pisos térmicos de Colombia. Revista de Investigación Agraria y Ambiental. RIAA 1: 31-35. DOI: https://doi.org/10.22490/2145645 $\underline{3.890}$

SEMARNAT [Secretaría de Medio Ambiente y Recursos Naturales]. 2015. Programa de Manejo Parque Nacional Cofre de Perote o Nauhcampatépetl. Secretaría de Medio Ambiente y Recursos Naturales, Comisión Nacional de Áreas Naturales Protegidas. México. https://simec.cona np.gob.mx/pdf_libro_pm/112 libro_pm.pdf (accessed April 1, 2020)

Silva ADA, Pinho DB, Costa H, Pereira OL, Lopes UP. 2014. First report of leaf spot caused by Myrothecium roridum on Coffea canephora in Brazil. Plant Disease
98: 1587. DOI: https://doi.org/10.1094/PDIS-06-14-0643 $\underline{-P D N}$

SAS [Statistical Analysis System]. 2003. SAS Institute, Cary, NC, Version 9 https://www.sas.com (accessed Septiembre 18, 2019).

Strobel G, Daisy B. 2003. Bioprospecting for microbial endophytes and their natural products. Microbiology and Molecular Biology Reviews 67: 491-502. DOI: https:// doi.org/10.1128/MMBR.67.4.491-502.2003

Vázquez J. 2014. Fenología reproductiva de las comunidades vegetales del Parque Nacional Cofre De Perote, Veracruz, México. MSc. Thesis. Universidad Veracruzana.

Vera ML. 1995. Efecto de la altitud en la fenología de la floración en especies arbustivas del norte de España. Lagascalia 18: 3-14.

Verdi G, Brighente C, Pizzolatti G. 2005. Gênero Baccharis (Asteraceae): aspectos químicos, econômicos e biológicos. Química Nova 28: 85-94. DOI: http:// dx.doi.org/10.1590/S0100-40422005000100017

Williams-Linera G, Meave J. 2002. Patrones fenológicos. In: Guariguata M., Kattan G. eds. Ecología y Conservación de Bosques Neotropicales. San José, Costa Rica. Libro Universitario Regional, pp. 407- 431. ISBN: 109968801119

Zar J. 2010. Biostatistical Analysis. Unites States of America: Pearson Prentice Hall. ISBN: 978-0-13-100846-5

Ziello C, Estrella N, Kostova M, Koch E, Menzel A. 2009. Influence of altitude on phenology of selected plant species in the Alpine region (1971-2000). Climate Research 39: 227-234. DOI: https://doi.org/10.3354/ $\underline{\mathrm{cr} 00822}$

\footnotetext{
Associate editor: Miguel Olvera Vargas

Contribuciones de los autores: MGRG, diseño experimental, trabajo de campo, trabajo de laboratorio, análisis de datos, redacción; FACP, financiamiento, análisis de redacción; MRPL, financiamiento, análisis de redacción; OBR, trabajo de laboratorio; EAG, diseño experimental, análisis de redacción; LRSV, idea original, financiamiento, diseño experimental, análisis de datos, redacción.
} 